\title{
Individual payments as a longer-term incentive in online panels
}

\author{
AnJa S. Göritz and Hans-Georg WolfF \\ University of Erlangen-Nuremberg, Nuremberg, Germany \\ AND \\ DaNiel G. Goldstein \\ London Business School, London, England
}

\begin{abstract}
Does it pay to pay online panel members? A three-wave longitudinal experiment was conducted with an online panel to examine whether per person payments, paid through an online intermediary, influence response and retention rates. In the payment condition, participants were promised payment for participation at each wave, whereas control participants were not offered any payment. The promise of a payment had a negative effect on response in Wave 1, but a positive effect on response in Wave 2. Payment had no significant effect on retention. Completing a given wave was an indicator for responding to a subsequent invitation.
\end{abstract}

For many research projects, Web-based data collection is methodologically and economically advantageous, especially if it is carried out in so-called online panels. An online (access) panel is a pool of registered people who have agreed to occasionally take part in Web-based studies. Because of the benefits of online panels, such as short field times, availability of panelists' historical and profile data, potential to obtain large samples, and ethical advantages (for a more detailed discussion, see Göritz, 2007), the bulk of all Web-based research is conducted in online panels (Batinic \& Moser, 2005; Couper, 2000).

In view of the importance of online panels for data collection, it is vital to develop methods for promoting the quality of data that are gathered with them. Data quality is a multifaceted construct (see Bailar, 1984; de Kampen, 2006; de Leeuw, 1992). It is characterized by the absence of both measurement error and nonresponse error (Dillman \& Bowker, 2001; Groves, 1989). Measurement error refers to the inaccuracy of responses due to the measurement instrument, the measurement situation, or aspects of the respondent's behavior. Nonresponse in its many forms, such as refusal to respond to a study request, the premature abandonment of a study, or the omission of items, reduces the effective sample size and, thus, the precision of parameter estimates in substantive analyses. If nonresponse occurs systematically (i.e., respondents and nonrespondents differ with regard to variables that are measured in the study) and is not taken into account, the study outcome is biased (Groves \& Peytcheva, 2008; Stoop, 2005).

By providing incentives for participating in a study, researchers can influence panelists' likelihood of taking part in studies, as well as the quality of the panelists' responses. Because employing incentives incurs costs in terms of money and manpower, we need to know its impact on data quality. In this study, the primary focus was nonresponse. In particular, we were interested in whether per capita payment would increase response and retention in online panel studies when used as a longer term incentive. In the online context, response rate equals the number of panelists who arrived at the first Web page of a study, divided by the number of panelists who had been e-mailed invitations to the study. Retention rate equals the number of panelists who stay until the last page of a study, divided by the number of responders.

The focus of previous research has been the one-time effect of incentives. However, because respondents in online panels are requested to participate in studies repeatedly, the longer term effect of incentives is of greater interest. Results from cross-sectional studies may not generalize to longitudinal or repeated experiments because, in such studies, participants gain experience with the task that is likely to affect the decision whether to participate in a subsequent wave or survey. For example, participants may remember whether they took part in previous waves, whether a study was tedious to complete, and whether they liked the incentive (see, e.g., Hill \& Willis, 2001).

To our knowledge, there are two longitudinal experiments that have examined the impact of incentives in online panels: First, in a five-wave study, one group of panelists was sent a prepaid gift, whereas the other group received nothing (Göritz, 2008). Then, for their participation in the studies, half of the panelists were repeatedly offered redeemable loyalty points, and the others were offered inclusion in cash lotteries. At the outset of the series of waves, the prepaid gift significantly increased participation ( $84.8 \%$ vs. $79.4 \%)$. The gift was especially

A. S. Göritz, anja.goeritz@psychologie.uni-wuerzburg.de 
useful when combined with the lottery. However, the effects of the prepaid gift and its combination with the lottery faded throughout the waves of the study. Moreover, initially, there was no difference in participation between people offered loyalty points and those offered the lottery. Over time, however, loyalty points became more attractive relative to the lotteries. Furthermore, the pattern of participation in this study followed a Markov chain (see, e.g., Brennan \& Hoek, 1992; Hagenaars, 1990), indicating that participants who had responded at a given wave were more likely to respond in subsequent waves.

Second, in a four-wave experiment, Göritz and Wolff (2007) offered panelists either repeated inclusion in a gift certificate lottery or no incentive at all. Independent of the lottery, panelists who had responded in a given wave were more likely to respond in the next wave. Thus, there was a Markov process at play again. The lottery had a direct positive effect on response for the first wave $(67.7 \%$ vs. $55.0 \%$ ), but not for the next wave. The positive effect of the lottery on response at the initial wave was carried over into later waves, mediated by the Markov process. The lottery had no effect on dropout. Furthermore, dropout in a given wave was a reliable predictor of refusal to participate in the subsequent wave.

The scarcity of longitudinal studies aside, previous research has focused primarily on lotteries (Göritz, 2006b) and has neglected other feasible incentives, such as per capita payments. This is surprising, given the ineffectiveness of cash lotteries when they are used in online panels repeatedly (Göritz, 2006a), which is mirrored by results from offline surveys in which lotteries generally result in lower response rates than do individual payments (Dillman, 2000; Hubbard \& Little, 1988). In the present study, we focus on per capita payments. Specifically, participants are promised to receive a certain amount of money for taking part in a survey. Several meta-analyses of offline surveys have shown that postpaid monetary incentives are effective (Edwards et al., 2002, $d=0.10$; Yu \& Cooper, $1983, d=0.10)$. Church (1993) found that postpaid monetary incentives increased response $(d=0.09)$, although not significantly. For practical reasons, individual payments in Web surveys are usually carried out via specialized intermediaries, such as PayPal (Göritz, in press). In PayPal, an option called mass payment makes it possible to pay a large number of recipients simultaneously.

To our knowledge, Bošnjak and Tuten (2003) have, to date, conducted the only experiment examining per capita payments via $\mathrm{PayPal}$ - albeit in a cross-sectional approach, and not in an online panel. In a survey of members of a professional association, Group 1 was prepaid US\$2 via PayPal within the first contact, Group 2 was promised to receive US\$2 via PayPal on completion of the survey, Group 3 was offered to be entered into a cash lottery on completion of the survey, and Group 4 was the control group. The lottery significantly increased Group 3's response rate (35.9\%) in comparison with those of Groups 1, 2, and 4 (25.6\%, 27.3\%, and 26.6\%, respectively). With regard to completion rates, which pertain to both the skipping of survey questions and to dropout, only the difference between the lottery group $(65.3 \%)$ and the control group (48.3\%) reached significance. Bošnjak and Tuten attributed the unexpected ineffectiveness of the per capita payment to the fact that obtaining a payment in the context of an online study does not compare to receiving cash offline, because an electronic payment via an intermediary is not the same as money in one's hand.

To find out whether online payments would increase response and retention rates, we conducted a longitudinal experiment in an online panel. In line with Bošnjak and Tuten (2003), we did not expect an effect on response and retention in the first survey wave. Retrieval of an electronic payment requires technical knowledge on the part of the respondent, as well as registration with the intermediary. Thus, in relation to a respondent's Web literacy, this process can be more or less cumbersome and possibly risky in terms of data security, which could make electronic payment less appealing. However, once respondents have had experience with electronic payments, they are almost as comfortable collecting money via this intermediary as they are collecting cash. Experience with electronic payments in the first wave means that respondents will have already overcome the obstacle of registering with the intermediary, which in turn suggests that they may have overcome their qualms about data security or that they did not have such fears in the first place.

For our examination of the effects of such payment on response and retention across multiple survey waves, we formulated a number of hypotheses. These hypotheses are described below.

Hypothesis 1A. In the first survey wave, panelists who are offered an electronic payment are equally likely to respond to the study request as are those who have not been offered an incentive.

Hypothesis 1B. In the first survey wave, panelists who are offered an electronic payment are equally likely to be retained until the end of the study as those who have not been offered an incentive.

Hypothesis 2A. In later survey waves, panelists who are offered an electronic payment are more likely to respond to the study request than are control participants.

Hypothesis 2B. In later survey waves, panelists who are offered an electronic payment are more likely to be retained until the end of the study than control participants.

The effect of the payment on response and retention aside, two additional hypotheses examine participation across the waves of the survey, independent of the payment.

Hypothesis 3. Retention across the waves follows a Markov process. That is, people who stay until the final page of the questionnaire in a given wave are more likely to stay until the final page in the next wave.

Hypothesis 4. Respondents who are retained at a given wave are more likely to respond at the next wave than are respondents who drop out at a given wave.

\section{METHOD}

\section{Sample and Procedure}

A three-wave experiment was conducted in September, 2005. The topic of the survey was voting behavior. We sent e-mail invitations to a sample of 496 members of a university-based online panel. The participants' mean age was 33.8 years $(S D=10.9)$, and $45 \%$ were 
Table 1

Response, Retention, and Net Usable Data With and Without Payment, per Wave

\begin{tabular}{|c|c|c|c|c|c|c|c|c|c|}
\hline & \multicolumn{3}{|c|}{ With Payment } & \multicolumn{3}{|c|}{ Without Payment } & \multicolumn{3}{|c|}{ Total } \\
\hline & Responders & $n$ & $\%$ & Responders & $n$ & $\%$ & Responders & $n$ & $\%$ \\
\hline \multicolumn{10}{|c|}{ Response } \\
\hline W1 & 91 & 250 & 36.4 & 111 & 246 & 45.1 & 202 & 496 & 40.7 \\
\hline W2 & 73 & 91 & 80.2 & 66 & 111 & 59.5 & 139 & 202 & 68.8 \\
\hline W3 & 56 & 72 & 77.8 & 55 & 66 & 83.3 & 111 & 138 & 80.4 \\
\hline \multicolumn{10}{|c|}{ Retention } \\
\hline W1 & 85 & 91 & 93.4 & 102 & 111 & 91.9 & 187 & 202 & 92.6 \\
\hline W2 & 60 & 73 & 82.2 & 52 & 66 & 78.8 & 112 & 139 & 80.6 \\
\hline W3 & 49 & 56 & 87.5 & 45 & 55 & 84.7 & 94 & 111 & 84.7 \\
\hline \multicolumn{10}{|c|}{ Usable Data } \\
\hline W1 & 85 & 250 & 34.0 & 102 & 246 & 41.5 & 187 & 496 & 37.7 \\
\hline W2 & 60 & 250 & 24.0 & 52 & 246 & 21.1 & 112 & 496 & 22.6 \\
\hline W3 & 49 & 250 & 19.6 & 45 & 246 & 18.3 & 94 & 496 & 19.0 \\
\hline
\end{tabular}

female. Completion of each wave took about 10 min. Participants were randomly assigned to one of two conditions. In the control condition $(n=246)$, panelists did not receive any information about the payment. In the payment condition $(n=250)$, panelists were promised to be paid $€ 1.50$ via PayPal (roughly US\$1.85) for participation in a wave. This assignment was kept constant over the three waves. This sample had not been offered a payment via PayPal in this panel before. Panelists in the payment condition were informed that if they did not have a PayPal account they would need to register with PayPal (for free) to receive the money, unless they wanted to take part without any remuneration, which was welcome as well.

Note that the study was not announced as a longitudinal study at the outset. Instead, in the second wave (W2) - without prior noticepanelists were told that this was a follow-up to a study in which they had participated about 2 weeks ago; in the third wave (W3), they were told that this was the final part of a study in whose first and second parts they had recently taken part. Only people who had responded in a given wave were invited to the next wave. Eligible respondents received the first PayPal payment of $€ 1.50$ before they were invited to $\mathrm{W} 2$. Because there were only a few days between $\mathrm{W} 2$ and $\mathrm{W} 3$, eligible participants received their second payment after W3 (i.e., $2 \times € 1.50$ if they had taken part in both W2 and W3, or $€ 1.50$ if they had taken part in W2 only). Response at Wave 1 (W1) was independent of age and sex (all $p$ s $>.30$ ). In further analyses, we included sex and age into the log-linear path models reported in the Results section. Since neither variable had a significant impact or changed the interpretation of our results, we report the analyses without sex and age.

\section{Analyses}

We used log-linear path models (Hagenaars, 1990; Vermunt, 1997b) to analyze the effects of the payment on response and retention at the three waves. These models enabled us to examine relations among several categorical variables in a single modeling approach. Log-linear path models can also account for the missing data structure of the present data. Our data have a monotone structure of missing data (Fay, 1986); panelists who did not respond to a given wave have missing data on the retention variable at this wave.

To test our hypotheses, we examined several models in a hierarchical manner. Paths were sequentially added or deleted, depending on the improvement in model fit (Hagenaars, 1990). We used conditional likelihood ratio tests and the Bayesian information criterion (BIC; Raftery, 1995) to evaluate model fit. We used LEM (Vermunt, 1997a) to analyze the data.

We also tested whether the payment had an effect on other facets of data quality - that is, skipping of one item where an answer was optional, length of the answer to one open-ended question, discrepancy between panelists' sex and age (according to the profile and questionnaire data), extremely long or short questionnaire completion times, and response styles, with three items. Data quality according to these criteria was very high, and no systematic differences between the payment and the control group were discernible, even when we combined the different criteria into an index of data quality. In the interest of brevity, we will not report analyses on these other facets of data quality in more detail.

\section{RESULTS}

Table 1 shows response, retention, and usable data in the two experimental conditions across the three waves. The response rate was lower in the payment condition at W1 $\left(36.4 \%\right.$ vs. $\left.45.1 \% ; \chi^{2}(1)=3.91, p<.05\right)$, but no consistent pattern emerged over all three waves. Retention was consistently higher in the payment condition. The share of usable data that was produced by invitees who stayed until the end of the questionnaire is the same (i.e., about 19\%) in both conditions at W3.

In order to test the hypotheses, we examined 14 loglinear path models. These models included paths from payment to response and retention at all waves. Some models included coefficients that were constrained to be equal and others included coefficients that were not constrained.

In the interest of brevity, we will not present all of the intermediate models, but will focus on the final model and the substantive results of the tests. The complete results with intermediate modeling steps are available from the first author upon request. Figure 1 depicts the final model, which has an adequate fit $[\operatorname{LR}(13)=13.15, p=.43]$. The path coefficients $B$ in Figure 1 are $\log$ odds ratios. A coefficient $B<0$ reflects a negative effect, whereas $B>0$ reflects a positive effect.

With regard to Hypothesis $1 \mathrm{~A}$, there was an unexpected negative effect of the payment on response at W1 $(B=$ $-0.36, S E=0.18, z=1.97, p<.05)$, in that panelists to whom a payment was offered were less likely to respond to the first study wave. Since many people find odds ratios more easy to interpret than $B$, reversing the logarithm by $e^{-0.36}$ yields an odds ratio of 0.70 . The odds ratio of 0.70 shows that the ratio of responders to refusals is only 0.70 times as high when a payment is promised as it is in the control condition. Hypothesis $1 \mathrm{~B}$ was retained, because there was no effect of the payment on retention at $\mathrm{W} 1 \mathrm{(} B=$ $-0.22, S E=0.55, z=0.41, p=.68)$. This path is therefore not included in the final model shown in Figure 1.

In Hypothesis 2A, we postulated a positive effect on response at later waves. In partial support of Hypothesis 2A, 


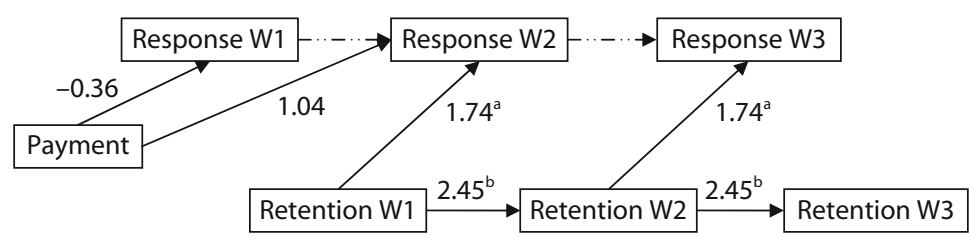

Figure 1. Effects of repeated payments on response and retention across three survey waves (final model with path coefficients). Coefficients with identical superscripts are restricted to equality.

we found a significant positive effect of payment on response at W2 $(B=1.04, S E=0.34, z=3.11, p<.01$; odds ratio $=2.77)$, but not at $\mathrm{W} 3(B=-0.55, S E=0.46$, $z=1.20, p=.23)$. In $\mathrm{W} 2$, the ratio of responders to refusals is 2.77 times as high when a payment is promised as it is in the control condition. We did not find any significant effects of payment on retention at later waves, yielding no support for Hypothesis $2 \mathrm{~B}$ (all $p \mathrm{~s}>.20)$. Therefore, these paths were dropped from the final model.

In Hypothesis 3, we posited that retention would follow a Markov process; that is, individuals who were retained at a given wave should be more likely to be retained at a later wave. The effect of retention at $\mathrm{W} 1$ on retention at $\mathrm{W} 2$ was positive, but marginally significant $(B=1.48, S E=1.02$, $z=1.45, p=.07$, one-tailed). The effect of retention at $\mathrm{W} 2$ on retention at $\mathrm{W} 3$ was significant $(B=2.80, S E=$ $0.64, z=4.36, p<.01$, one-tailed). In a more parsimonious model, we restricted these two effects to equality. The resulting path coefficient was significant $(B=2.45, S E=$ $0.55, z=4.48, p<.01$, one-tailed; odds ratio $=11.59)$, whereby the model with the equality restriction obtained a better fit $(\mathrm{BIC}=1,031.0)$ than did the model with two separate effects $(\mathrm{BIC}=1,036.0)$. Therefore, Hypothesis 3 is supported. Among individuals who were retained at a given wave, the ratio of retainees to dropouts in the next wave was 11.6 times that ratio among individuals who dropped out at a given wave.

In Hypothesis 4, we predicted that retention at a given wave would be positively related to response at the subsequent wave. This hypothesis was confirmed by two significant path coefficients of retention at W1 to response at $\mathrm{W} 2$ ( $B=2.03, S E=0.63, z=3.25, p<.01$, one-tailed), and from retention at $\mathrm{W} 2$ to response at $\mathrm{W} 3(B=1.57$, $S E=0.47, z=3.32, p<.01$, one-tailed). Again, we examined whether restricting these two effects to equality improved model fit. The restricted effect remained significant ( $B=1.74, S E=0.37, z=4.70, p<.01$, one-tailed; odds ratio $=5.70)$, and this more parsimonious model obtained a better fit $(\mathrm{BIC}=1,031.0)$ than did the model with two unrestricted coefficients $(\mathrm{BIC}=1,036.8)$. Thus, retention at a given wave is related to response at a subsequent wave as hypothesized: The ratio of responders to refusers was 5.7 times as high for individuals who were retained in the previous wave as it was for those who dropped out.

\section{DISCUSSION}

We have presented a longitudinal experiment on the effectiveness of electronic payment via an online intermedi- ary (i.e., PayPal) as an incentive for study participation in online panels. In Hypothesis $1 \mathrm{~A}$, we posited that a payment via PayPal would have no effect on response in the initial wave. This hypothesis was not supported. Instead, using electronic payment as an incentive for the first time had the surprising effect of lowering response rates and thereby increasing research costs. Because this was the first time that this sample was offered a payment via PayPal, unfamiliarity with the procedure could explain the lack of effectiveness of the payment. However, not knowing or not being used to PayPal or minding the effort it takes to collect this payment (see also Bošnjak \& Tuten, 2003) cannot explain the negative effect of the PayPal payment, especially given that people in the payment condition were also given the opportunity to take part without remuneration.

Two categories of reasons could account for the payment's deleterious effect. First, although we avoided alluding to the merits of the commercial intermediary (PayPal), some panelists - especially if they had just skimmed the e-mail invitation - could still have thought that the panel was endorsing PayPal, a for-profit organization. Despite the fact that creating a PayPal account and receiving money is free of charge, we could not conduct our experiment without promoting PayPal to some degree: This is unavoidable when one is working with any branded commercial service. The promotion of a for-profit company could have given panelists pause if they had not explicitly signed up for this. If this were true, however, other incentives involving a commercial provider, such as Amazon gift certificates, should be deleterious as well. Further experiments can tease apart whether panelists' dislike is generalized to the promotion of any kind of for-profit company or restricted to sensitive areas, such as financial services, about which Internet users are often apprehensive (Biswas \& Biswas, 2004). Moreover, future experiments could clarify whether such a dislike is specific to (or exacerbated by) noncommercial panels, such as the one at hand, or whether members of any online panel, including a commercial market-research panel, resent the promotion of a commercial company.

The second category of reasons for the payment's deleterious effect pertains to intrinsic versus extrinsic motivation. It has been suggested that payment can reduce people's interest in taking on work, or it can diminish the quality of their achievements (Deci, 1971). Payment could corrupt a person's intrinsic motivation by degrading to the level of commercial exchange what he or she intends to be a generous service. In a similar vein, the offer of a payment could cause people to question whether the 
money actually compensates them for their efforts (Dillman, 1978), whereas being asked to participate without incentive appeals to goodwill and altruism. However, this explanation is limited to noncommercial panels; Bošnjak and Tuten (2003) did not observe a deleterious effect of payment, and a deleterious effect has not surfaced in metaanalyses, either (e.g., Church, 1993; Edwards et al., 2002; Yu \& Cooper, 1983). Indeed, in noncommercial panels, the motivation to help in research is likely stronger than in commercial panels.

In Hypothesis 1B, we posited that payment has no effect on retention in the first wave. Similar to Bošnjak and Tuten (2003), we found the effect of the payment on retention at Wave 1 not to be statistically significant, as predicted. In terms of effect magnitude, this effect was tiny, at $w=0.01$. If - as according to Cohen (1987) - the effect had been small or medium, we would have had acceptable levels of power to detect that effect: The power to detect a small difference $(w=0.1)$ at $N=496$ and $\alpha=.05$ was 0.60 , and the power to detect a medium effect $(w=0.3)$ was 0.99 . Thus, we do not think that the nonsignificance of our result was due to low power.

In contrast to the first wave, in the second wave, panelists who were offered money were more likely to respond than were control participants (Hypothesis 2A). Because participants had already been paid for their participation in the initial wave, they were familiar with the process of collecting money via PayPal and had learned that the panel's promise of electronic payment was kept. Moreover, being invited to the second wave applied only to people who had responded to the first wave; that is, it involved only those whom the payment did not prevent from responding in the past.

In the third wave, there was no difference in response between paid and control respondents. Note that when participants were invited to wave three, the payment was still owed. Thus, the possible response-enhancing effect of the promised payment could have been offset by the participants' disapproval of not having been paid the money from the last wave before being invited to a new wave. That said, participants were informed that it would take some weeks to receive payment, so trust was not violated. To find out whether this conjecture holds, an experiment is needed where payments come immediately after each wave.

In Hypothesis 2B, we posited that in later survey waves, panelists who were offered a payment would more likely be retained than would control participants. Although the payment did have a mild net-positive effect on retention in the second and third waves, this tendency did not reach statistical significance. A positive effect on retention might be more pronounced when surveying a less motivated sample or if respondent burden is high. In the case at hand, a ceiling effect due to a high degree of retention among control participants seems to have occurred. Studies are needed to establish that payments really do not motivate respondents to stay until the end of the questionnaire in later survey waves. Such studies must be designed to have high power for small effect sizes, so that low power can be eliminated as a possible reason for retaining the null hypothesis. It would be surprising if payments did not motivate respondents to be retained in later survey waves given that (1) by the time they reach the later waves, most respondents should be familiar with redeeming electronic payments, and (2) the payment acts on respondents who chose to respond, knowing about the payment terms.

In summary, the harmful effect of payment on response in the initial wave was increasingly reduced over the remaining waves by its positive effect on response in Wave 2 and its mildly beneficial (but not significant) effect on retention in each wave. After conclusion of this three-wave study, the number of usable responses was comparable in the control and in the payment condition; however, more money had been spent in the payment condition. It is conceivable that with studies of more than three waves, PayPal payments would start to pay off in terms of more usable data sets. However, at this stage, this is a conjecture beyond any data. Moreover, the PayPal payment did not influence facets of data quality other than response and retention. That said, a PayPal payment could have an impact on facets of data quality not examined in these studies, such as the internal consistency when filling out psychometric scales, or response nondifferentiation in grid questions (Göritz, 2005).

With regard to response and retention across the waves (irrespective of the payment), Hypotheses 3 and 4 were supported. Replicating the results of many other longitudinal studies, the present study showed that people who stayed until the end of a given wave were more likely to stay until the end of the subsequent wave. We replicated a finding of Göritz and Wolff (2007) that dropout at an earlier wave is an early indicator for nonresponse at the next wave. Survey managers could act on this information. Perhaps they can avert participants' refusal by strengthening trust through personal communication, offering technical help, or offering a refusal conversion incentive.

The present study has limitations that point to research questions to be addressed in the future. Since this experiment was the first longitudinal experiment in which a PayPal payment was used as an incentive, the stability of the findings should be established in a similar study, but with higher power. Moreover, given that the experiment at hand was conducted in a university-based panel, it would be worthwhile to conduct similar experiments in commercial online panels. Because market-research panelists are probably more business-minded than volunteers in a not-for-profit panel, they might be less irritated by the panel's collaboration with a commercial intermediary such as PayPal, or they might be more expecting of payment in general. Therefore, a PayPal payment might start to pay off after fewer survey waves than in a not-for-profit panel. In addition, since attitudes toward risk (concerning nonpayment or data security) may differ by culture, extending the analysis to other countries that are just beginning to deal with online payments would provide a test of robustness. In addition, it would be interesting to conduct similar experiments with different treatment doses. Without it necessarily being a linear relationship, we would expect that the higher the sum offered, the quicker and stronger the reduction of nonresponse.

Given the initially adverse effects of the PayPal payment in the present study, it might be worthwhile to try other 
forms of per capita payment. For example, many Germans are comfortable with providing bank details for making and receiving payments (which comes as a surprise to many North American ears). Therefore, participants could be asked their bank details and receive money directly into their bank accounts. For researchers and survey managers, the allocation of the money would be as easy as a mass payment through PayPal, because many banks accept electronic files of pay orders to effect bulk payments. Providing bank details might not work well in other countries, where people are reluctant to disclose this information, so alternative payment options merit examination.

We have addressed some of these open issues in an experiment parallel to the one presented here (Göritz, Wolff, \& Goldstein, 2008). The two experiments intersect, in that they were conducted in the same university-based online panel, with comparable (but nonoverlapping) samples from all walks of life, on a similar topic, and with invitees who were offered an electronic payment for the first time since they had become panel members. The experiments differ in that the study at hand was not announced to be a longitudinal study at the outset, and online payments were promised for participation in each study wave. By contrast, the experiment by Göritz et al. was described as a longitudinal study from the beginning, and electronic payment was promised to be made once after participation in the study's two waves. The high degree of correspondence of results in the overlap of the two experiments suggests that the findings from the experiment at hand are robust.

In sum, when online payments are used for the first time in an online panel, they can have the surprising effect of lowering response rates. In the rapidly evolving environment of online experimentation, it may pay to listen more to evidence than to intuition in making decisions about incentives.

\section{AUTHOR NOTE}

This work was supported by a University of Erlangen-Nuremberg postdoctoral scholarship (HWP) to the first author. Correspondence concerning this article should be addressed to A. S. Göritz, Work and Organizational Psychology, Würzburg University, Röntgenring 10, 97070 Würzburg, Germany (e-mail: anja.goeritz@psychologie.uni-wuerzburg.de).

\section{REFERENCES}

BaILar, B. A. (1984). The quality of survey data. Proceedings of the Section on Survey Research Methods of the American Statistical Association, 43-52.

Batinic, B., \& Moser, K. (2005). Determinanten der Rücklaufquote in Online-Panels [Determinants of response in online panels]. Zeitschrift für Medienpsychologie, 17, 64-74.

Biswas, D., \& Biswas, A. (2004). The diagnostic role of signals in the context of perceived risks in online shopping: Do signals matter more on the Web? Journal of Interactive Marketing, 18, 30-45.

BošnJaK, M., \& Tuten, T. L. (2003). Prepaid and promised incentives in Web surveys: An experiment. Social Science Computer Review, 21, 208-217.

Brennan, M., \& HoeK, J. (1992). The behavior of respondents, nonrespondents, and refusers across mail surveys. Public Opinion Quarterly, 56, 530-535.

Church, A. H. (1993). Estimating the effect of incentives on mail survey response rates: A meta-analysis. Public Opinion Quarterly, 57, 62-79.

COHEN, J. (1987). Statistical power analysis for the behavioral sciences (2nd ed.). Hillsdale, NJ: Erlbaum.
COUPER, M. P. (2000). Web surveys: A review of issues and approaches. Public Opinion Quarterly, 64, 464-494.

DeCI, E. L. (1971). Effects of externally mediated rewards on intrinsic motivation. Journal of Personality \& Social Psychology, 18, 105-115.

DE KAMPEN, J. K. (2006). The impact of survey methodology and context on central tendency, nonresponse and associations of subjective indicators of government performance. Quality \& Quantity, 41, 793-813.

DE Leeuw, E. D. (1992). Data quality in mail, telephone, and face to face surveys. Amsterdam: TT-Publikaties.

Dillman, D. A. (1978). Mail and telephone surveys: The total design method. New York: Wiley.

Dillman, D. A. (2000). Mail and Internet surveys: The tailored design method (2nd ed.). New York: Wiley.

Dillman, D. A., \& Bowker, D. K. (2001). The Web questionnaire challenge to survey methodologists. In U.-D. Reips \& M. Bošnjak (Eds.), Dimensions of Internet science (pp. 159-178). Lengerich, Germany: Pabst.

Edwards, P., Roberts, I., Clarke, M., Diguiseppi, C., Pratap, S., Wentz, R., \& KWAN, I. (2002). Increasing response rates to postal questionnaires: Systematic review. British Medical Journal, 324, $1183-1185$.

FAY, R. E. (1986). Causal models for patterns of nonresponse. Journal of the American Statistical Association, 81, 354-365.

GöRITZ, A. S. (2005). Contingent versus unconditional incentives in WWW-studies. Metodološki Zvezki-Advances in Methodology \& Statistics, 2, 1-14.

Göritz, A. S. (2006a). Cash lotteries as incentives in online panels. Social Science Computer Review, 24, 445-459.

GöRITZ, A. S. (2006b). Incentives in Web studies: Methodological issues and a review. International Journal of Internet Science, 1, 58-70.

GöRITZ, A. S. (2007). Using online panels in psychological research. In A. N. Joinson, K. Y. A. McKenna, T. Postmes, \& U.-D. Reips (Eds.), The Oxford handbook of Internet psychology (pp. 473-485). Oxford: Oxford University Press.

Göritz, A. S. (2008). The long-term effect of material incentives on participation in online panels. Field Methods, 20, 211-225.

GöRITZ, A. S. (in press). Incentives in Web-based research. In S. Gosling \& J. A. Johnson (Eds.), Advanced methods for behavioral research on the Internet. Washington, DC: American Psychological Association.

GöRITZ, A. S., \& WoLFF, H.-G. (2007). Lotteries as incentives in longitudinal Web studies. Social Science Computer Review, 25, 99-110.

Göritz, A. S., WolfF, H.-G., \& Goldstein, D. G. (2008). Payments via Paypal as an incentive in longitudinal Web-based studies. Manuscript submitted for publication.

Groves, R. M. (1989). Survey errors and survey costs. New York: Wiley.

Groves, R. M., \& Peytcheva, E. (2008). The impact of nonresponse rates on nonresponse bias: A meta-analysis. Public Opinion Quarterly, 72, 167-189.

HagenaARs, J. A. (1990). Categorical longitudinal data: Log-linear panel, trend, and cohort analysis. Newbury Park, NY: Sage.

HILl, D. H., \& Willis, R. J. (2001). Reducing panel attrition: A search for effective policy instruments. Journal of Human Resources, 36, 416-438.

Hubbard, R., \& Little, E. L. (1988). Promised contributions to charity and mail survey responses: Replication with extension. Public Opinion Quarterly, 52, 223-230.

RAFTERY, A. E. (1995). Bayesian model selection in social research. Sociological Methodology, 25, 111-163.

Stoop, I. A. L. (2005). The hunt for the last respondent: Nonresponse in sample surveys. The Hague: Transaction Publishers.

VERMUNT, J. K. (1997a). LEM 1.0: A general program for the analysis of categorical data. Tilburg, The Netherlands: Tilburg University. Accessed September 2008 at http://spitswww.uvt.n1/ vermunt/.

Vermunt, J. K. (1997b). Log-linear models for event histories. Thousand Oaks, CA: Sage.

YU, J., \& COOPER, H. (1983). A quantitative review of research design effects on response rates to questionnaires. Journal of Marketing Research, 20, 36-44.

(Manuscript received April 19, 2008; revision accepted for publication June 19, 2008.) 